\title{
Spanning boundaries, transforming fields: The impact of community networks in scholarly communications
}

\author{
Katherine Skinner* \\ Educopia Institute, 1230 Peachtree Street, Suite 1900, Atlanta, GA 30309, USA
}

\begin{abstract}
This paper shares observations from the Educopia Institute about the impetus, process, and impact of building and sustaining targeted cross-sector collaborative networks. Based on more than 10 years of work with scholars, librarians, archivists, curators, and publishers in various fields, the paper opens with a quick overview of Educopia's community incubation model and the rationale for our explicit emphasis on cross-sector collaborative networks. It then shares case studies that illustrate how alignment serves as a tool to magnify the impact of investments made in the area of knowledge dissemination and scholarly communications. It closes with an overview of resources and a model Educopia Institute is developing to scale the lessons we've learned in community incubation that we believe will help more communities achieve the impact they seek.
\end{abstract}

Keywords: Scholarly communication, open access, community incubation, collaborative networks

\section{Introduction}

The Educopia Institute was founded in 2006 to provide community cultivation and incubation services to collaborative networks serving the library, archives, and museum sectors. At our core, Educopia is a network builder. To date, we have founded three affiliated communities that continue to live under the "Educopia Umbrella" - the Library Publishing Coalition, the MetaArchive Cooperative, and the BitCurator Consortium - and we have helped to build and grow more than a dozen additional networks in the U.S. and Canada, including the Digital Preservation Network (DPN), InDiPres, the Council of Prairie and Pacific University Libraries, and the Coalition to Advance Learning.

Educopia focuses on the structure, behavior, and evolving dynamics of collaborative networks. We want to help these groups succeed wildly in their varied attempts to effect change. To this end, Educopia's staff members engage in a range of activities - roughly grouped under the term "incubation." We work with communities at various stages, from inception to sun-setting, to help them make informed decisions on their governance and their organizational and business models. We also cultivate the volunteer leaders of such networks to help them actively grow both the network itself and the work/mission the network supports.

\footnotetext{
*Corresponding author. E-mail: katherine@educopia.org.
} 
Educopia serves as a kind of "community of communities," a network builder that also facilitates cross-talk between existing networks, both within and beyond Educopia. Our three current communities learn from each other's experiences, as well as from the experiences of other networks we are currently incubating (e.g., the Software Preservation Network or providing with consulting services).

\section{The power of lenses and perspectives}

Our work is informed by sociology and organizational psychology and other disciplines of study that have demonstrated that system-level change can be orchestrated through the deliberate work of stakeholders across a whole system. Most of us, when faced with decisions of any scale at work, think with two main "lenses" or perspectives - our individual perspective (What do I need? What do I stand to gain or lose? How might this advance my career or my ambitions?) and our institutional perspective (What does our organization need? What does our organization stand to gain or lose?).

The individual lens is necessarily limited and relatively short-sighted in its aims. Advancement along most individual career trajectories is influenced by our adherence to specific, organizational norms and our ability to get along with key people within our organization. As we progress, we often move up a chain of command, beginning as a staff member reporting up to a range of embedded managers and directors. Over time, we might become a manager of staff, of a department, of multiple departments, or of an organization. Our individual lens might lead us to pursue work at different organizations as we move up this career trajectory based on our interests, relationships, and workplace culture desires.

The institutional perspective or lens values often aligns well with the individual perspective. When we work with our organizational lenses on, we think about what's good for our own organization. That organizational lens almost always honors stasis; that is, we are rewarded for doing what is safe and expected rather than for what is new and path-breaking. Even in organizations that value innovation and entrepreneurship, these qualities tend to be carefully cultivated within "lab" environments or other contexts that protect the operational reality of the organization from ongoing change. Organizations require predictability in order to ensure their longevity. As such, they reward habituated workflows and operate within a relatively-restricted context. That context is rarely considered to be something the organization itself has the power to change.

The decisions we make even with just these two lenses or perspectives in mind may be both fraught and complex. We usually have to balance the needs of our own career against the needs of our institution and act accordingly. But these two lenses, the individual and institutional, are only parts of a greater whole the system(s) in which we are embedded. Engaging a system perspective or lens forces us to think about the big-picture - not only just our success as an individual or organization, but also about the health and sustainability of the extended environment in which we do our work.

The system lens requires us to think about more than just what we need or can do today. We have to think about what we need to do to ensure that we can do our jobs in the future. We have to think beyond our organizational affiliation and identify with those who work with us at a field level. The system is the bigger picture in which our work occurs. It includes not just our organization, not just our field, but all of those components that work together to make it possible for us to do our work. For example, the system of scholarly communications includes not just publishers, but also all of the stakeholders involved: librarians, authors, readers/viewers, sellers, data analysts, funders, technologists, and so on.

In order for any activity to have a lasting impact on the system, players from across the system must be involved - whether by accident or by design. Take for example a major development in scholarly 
communications that emerged several decades ago, the practice of bundling subscriptions, also known as the "Big Deal." [1] This innovation was inspired by a system-level crisis that was affecting all players within the scholarly communications market. Due to a range of factors that include the invention of the Xerox machine, disciplinary specialization and the resulting introduction of new societies and journals, a quick rise in scholarly output, and the shift from print-to-digital production, societies and other publishers found their market to be shrinking in terms of both individual and library-based subscriptions [2].

A "subscription spiral" infamously resulted, wherein libraries cancelled journals, and then publishers responded by increasing the price-per-subscription for journals, which then yielded more cancellations. The system was destabilized, and in that moment, an accidental innovation was introduced that all stakeholders in the system embraced (at least temporarily). One key publisher, Academic Press (AP), identified a source of government funding for electronic content and pitched a project. The UK's Higher Education Funding Council funded the AP with a multi-journal, multi-year license that provided all UK higher education establishments with "free" use access to all of AP's titles.

The new arrangement suited all stakeholders when it launched in 1996. The funder could cite the project as exemplifying a new national digital infrastructure; libraries and researchers experienced vastly increased access to research publications; and the publisher, AP, gained a multi-year funding stream and a relatively-easy mechanism for providing electronic access to all of its publications in a way that did not require difficult administrative technical restrictions (i.e., identity management) due to its national scale. Through bundling everything into one subscription, AP found that it could leverage its "all or nothing" status to break the cancellation cycle. This foundation for the "Big Deal" was not laid via intention, nor was it pushed onto the system. Instead, the bundled subscriptions - initially paid for by a funding agency on behalf of its national universities - provided a short-term win for individual institutions. Because the organizations involved benefitted from the project, few questioned the cost model that would effectively and disproportionately "trap" one player - the library. This system-change moment ultimately did not repair the underlying system-based problems - the rising numbers and costs of journals vs. the shrinking budgets of libraries [3]. It still changed the already destabilized system, setting in motion an arrangement that has now lasted for decades.

Stakeholder buy-in, the successful distribution and reception of an innovation, is a mandatory component of system-wide change. Imagine the different reception that might have occurred in this instance if each of the stakeholder groups focused not on the individual and organizational lenses that championed the innovation (increased access to research! Short-term win!) but instead looked at the long-term implications of the new funding model ("all or nothing" pricing, consolidation of the market, steep increases in the price of journals, steep declines in funds available to support any other form of scholarship).

Much of Educopia's work is designed to address system-level issues. We intentionally assemble crosssector, multi-stakeholder groups and cultivate points of alignment between them. As we do so, we lean heavily on several facilitation methodologies, including "Boundary-Spanning Leadership" (Center for Creative Leadership) and "Collective Impact" (FSG).

\section{Facilitating change}

As we facilitate groups of stakeholders from different arenas, we do so deliberately and with specific aims; for example, we assemble journalists, editors, publishers, researchers, librarians, archivists, technologists, press associations, lawyers, and content aggregators to address the digital news preservation of news sources by marginalized communities in our "News on the Margins" work; or we assemble 
software designers, programmers, engineers, publishers, artists, archivists, curators, and lawyers to address software sustainability together in the "Software Preservation Network."

Before we can span boundaries in such groupings, we first have to acknowledge and reinforce difference. That is deeply counter intuitive; it is also essential for multi-stakeholder, cross-sector engagement to take hold. We use the Center for Creative Leadership (CCL)'s "Boundary-Spanning Leadership" model to guide our facilitation efforts. This model emphasizes the need to begin by "buffering" boundaries; i.e., breaking stakeholders into their specific identity groups and asking them to reflect on what makes them unique and different within this assemblage. This process of managing boundaries provides a sense of security and identity to each stakeholder group, enabling them to first emphasize and reinforce what value they bring into the collaborative effort.

Once the boundaries have been acknowledged and stabilized, the stakeholder groups can begin to connect across to each other, building trust and developing together a sense of communal identity based on the aims that they share. As they deepen in their awareness of the range of skills and perspectives available across these stakeholder groups, they can begin to mobilize their efforts, pointing their ships in the same general direction and moving towards their shared goals. This alignment is pivotal and provides a foundation for additional levels of engagement where those are needed, including fostering interdependence and enabling the transformation of the system as a whole.

As we facilitate multi-stakeholder groups, we also rely on many of the principles of "Collective Impact," a methodology that swiftly rose to prominence in the social sector after the publication of a 2011 article by John Kania and Mark Kramer in the Stanford Social Innovation Review [4]. The Collective Impact model begins with the same general purpose as "Spanning-Boundaries" - to align the interests of diverse stakeholder groups. This method begins with the establishment of a "Common Agenda" or shared vision among all stakeholders. This vision cannot be a platitude, but rather needs to be both concrete and measurable; e.g., increasing the number of accessible historical news sources by marginalized communities by fifty percent in ten years.

Once this agenda is established, the Collective Impact initiative develops shared measurement frameworks to track incremental progress towards the goal. Each stakeholder group will engage in its own, sector-specific activities that contribute to reaching the goal, and these become "Mutually Reinforcing Activities" between all of the stakeholder groups. Continuing our example from above, in the "News on the Margins" community, mutually reinforcing activities might take on the following shape:

As libraries, archives, and museums identify the African American newspapers in their archives, data analysts analyze that information and identify how best to assemble a full run of a historic newspaper title across many collecting institutions. Funders provide resources to enable the digitization of the title, and publishers work to resolve rights issues with journalists and photographers. Aggregators provide a platform for curating and providing perpetual access to the content.

In this example, as in other Collective Impact efforts, each stakeholder's activities reinforce another's, moving the entire initiative towards its ultimate goal. As each stakeholder group makes and measures its own progress towards goals in support of the shared vision, a facilitator ensures that all other stakeholders are aware of this forward momentum. This feedback loop of "Continuous Communications" is coordinated by a "Backbone Support" group that also helps each stakeholder group to refine its goals as necessary to stay in synch with all others. In other words, the stakeholder-specific work is coordinated by the "Backbone Support" (in essence, a set of focused facilitators who provide the connective tissue for the endeavor) such that each group's work bears an explicit, reciprocal relationship with the work of the collective whole. 
The purpose behind this methodology is to encourage every player to change simultaneously, deliberately, and in concert with each other. These changes then reinforce each other at a system level, making it more likely that those changes will "stick."

We use these facilitation methods in tandem across much of our work, both in creating and incubating nascent collaborative networks and also in helping existing collaborative networks to grow and transition in healthy and sustainable ways. To accomplish this, we use Educopia's Community Cultivation Model, which we have built over more than a dozen years of community facilitation work.

\section{Community cultivation model}

Spanning boundaries and establishing a shared agenda and measurement apparatus are helpful techniques in a wide-range of community cultivation activities, ranging from start-up enterprises (in which we help shape a nascent, promising network) to transition work (helping existing networks expand and grow in healthy ways, including through purposeful vision, administrative, technical, fiscal, community engagement, and governance decisions).

Our work provides essential scaling of the support services that collaborative networks need as they move from one stage to another. We provide technical, administrative, financial, and legal support for the core business functions that collaborative communities need to build and sustain in order to stay healthy over time. In our model, we see four main stages of growth that most collaborative networks go through in a cyclical fashion: (1) Formation (and later, reformation), (2) Validation, (3) Acceleration, and (4) Transition, documented in more detail below. We have developed strategies and models to identify which stage(s) a collaborative network is in along six common vectors: (a) vision, (b) administrative infrastructure, (c) technical infrastructure, (d) fiscal model, (e) community engagement, and (f) governance. We have also developed strategies and models to help collaborative networks grow and transition through each of those stages over time.

\section{(re)FORMATION}

Key challenge: How do we foster a healthy community?

Focal Points: establish mission/vision, strategic goals, and assessments; pilot services/products; prioritize development steps; establish scaffolding for communications and administration; establish financial plan with diversified funding streams; foster community relationships; establish/grow leadership.

\section{VALIDATION}

Key challenge: How do we demonstrate our viability?

Focal Points: articulate mission/vision and goals to others; build/strengthen interdependence on services/products; refine communications and administration scaffolding; expand technical infrastructure to support a growing community; actively develop funding model and establish initial operational reserves; empower and credit community members' engagement; test/iterate service model(s) within broadening circles; test efficacy of need; engage leadership in practical aspects of growth.

\section{ACCELERATION}

Key challenge: How do we grow fast (or fail fast)?

Focal Points: engage in second-phase strategic planning (evaluate and, if necessary, recalibrate mission/vision/value, conduct SWOT and landscape scans, update strategic goals and assessments); expand community and increase service/product use; streamline onboarding; strengthen internal communications 
and tools; generate revenue to cover operational costs $+10 \%$ (reserves); engage the community to help broadcast services/products; monitor landscape for competitors; establish concrete roles for all leadership members including via subcommittees that attend to specific operations (communications, fiscal integrity, etc.).

\section{TRANSITION}

Key challenge: How should we change for continued impact?

Focal Points: identify service/product gaps and challenges; establish or solidify organizational structure (select appropriate host or organizational form, ensure smooth transitions of legal/fiscal/organizational systems and identity); evaluate new administrative, communications, and technical tools that can refine the operational foundation (e.g., CRM); generate revenue to cover operational costs and R\&D; establish operational reserves to cover at least eighteen months of operations; reinforce and deepen relationships (internal and external); mature governance framework and documentation.

\section{Community cultivation in action}

At Educopia, pairing these two methodologies (the Collective Impact Model and the Community Cultivation Model) provides us with a solid base for community action. Our collaborative networks benefit from the secure identity provided by "Spanning-Boundaries Leadership" facilitation, and building on this base, we are able to help diverse groups set common agendas, establish mechanisms for measuring progress towards goals, and we are able to provide the backbone support to help them stay connected and refine those goals throughout a project or program trajectory.

\section{Software preservation network}

One example of this work is the Software Preservation Network (SPN), a nascent network that is still in early formation stages (see: http://www.softwarepreservationnetwork.org/). The goal of this network is to bring attention to a problem shared by industry, academia, and libraries, archives, and museums: the persistence of the software we use to create and access most of the knowledge objects created today.

Over the last 18 months, we have worked closely with SPN's founders to build a solid initial, volunteeronly structure for its operations. We have also helped to identify funding partners to help drive this work forward (currently the Institute of Museum and Library Services, the Alfred P. Sloan Foundation and the Andrew W. Mellon Foundation), and we are currently assisting this network as it builds a business model with a mix of revenue streams that will include active tech-based services such as emulation, and that will also equip its members to tackle the thorny legal challenges and policy challenges they face together, system-wide.

The parties involved include gamers and artists, engineers and designers, curators and archivists, programmers and publishers! The fusion of these voices is not always easy - different drivers activate different pockets of stakeholders within the growing community. But with attention, we are steadily identifying ways in which the interests of these groups can align towards shared goals. The net result is that instead of these groups working in isolation with their limited resources, either building parallel infrastructure or competing infrastructures, they are working together to magnify the impact of investments made to support the sustainability of our digital records and artifacts. 


\section{Library publishing coalition}

The Library Publishing Coalition is a membership organization of more than seventy international libraries (see: https://librarypublishing.org/). These libraries undertake publishing - sometimes in concert with a University Press, and sometimes as a solo endeavor, especially on campuses that do not have University Presses.

This Coalition is a thriving community of players who lacked a "home base" in 2012 when the initiative was founded. These publishers attended conferences and participated in communities of publishers, including the Society of Scholarly Publishers and the Association of University Presses, but their work was largely considered to be a small rogue pocket of activity, not a concentrated and growing subfield of publishing.

The Coalition didn't found itself to isolate Library Publishing from other forms of publishing; instead, it did so to draw attention to and support the ongoing activities of libraries in this space, including some of the innovative work they are promoting. The Coalition has, from the beginning, deliberately interfaced with and welcomed a range of perspectives through strategic affiliate programming, including authors and students, data analysts and administrators, university presses and aggregators, editors and librarians. These groups working together are now exacting change, as is marked through increasing publication activities, both by individual libraries and also by library-press collaborations.

\section{Conclusion}

Spanning boundaries, sectors, and fields, we use Educopia's community cultivation model to unite people and institutions around shared challenges in digital preservation, scholarly communications, and continuing education. Our work helps organizations to work collectively to ensure that knowledge is sustainably produced, widely shared, and preserved. Our intentionally nimble team facilitates a wide-range of collaborative networks, assisting these groups with ongoing refinement and calibration of governance, business planning, communications, and technical operations. Through this work, we find that groups of institutions acting in concert across field and disciplinary boundaries accomplish more than any of the individual players could hope to do alone.

\section{About the Author}

Dr. Katherine Skinner is the Executive Director of the Educopia Institute, a not-for-profit educational organization that builds networks and collaborative communities to help cultural, scientific, and scholarly institutions achieve greater impact. In this capacity, she has founded and incubated three major communities (MetaArchive Cooperative, Library Publishing Coalition, and BitCurator Consortium) and has provided consulting services for dozens more (e.g., Digital Preservation Network, CLOCKSS, Software Preservation Network, the Coalition to Advance Learning). Skinner received her Ph.D. from Emory University. She has co-edited three books and has authored and co-authored numerous reports and articles. She is currently Principal Investigator for research projects on continuing education (Nexus, Mapping the Landscapes), digital preservation (Open Source Software Archival Workflows (OSSArcFlow), Preserving Electronic Government Information), and scholarly communication (Developing a Library Publishing Curriculum). She regularly teaches graduate courses and workshops in multi-stakeholder community 
management, digital librarianship and preservation topics, and provides consultation services to groups that are planning or implementing digital scholarship and digital preservation programs.

\section{References}

[1] R. Poynder, The big deal: Not price but cost, Information Today 28(8) (2011) http://www.infotoday.com/it/sep11/The-BigDeal-Not-Price-But-Cost.shtml (last accessed June, 11 2018)).

[2] K. Skinner, Taking action at a critical moment, Serials Review 41(3) 135-142 doi: 10.1080/00987913.2015.1069779.

[3] R. Poynder, op. cit.

[4] J. Kania and M. Kramer, Collective impact, Stanford Social Innovation Review, (2011) https://ssir.org/articles/entry/collective_impact (last accessed June 11, 2018). 